\title{
EXAMINING TRAINING PERFORMANCE OF TVET TRAINERS WITH/WITHOUT TVET CERTIFICATE IN THE REPUBLIC OF KOREA
}

\author{
Woo, H., Kim, W., Yi, Y. \& Yoon, G. \\ Graduate School of Human Resource Development \\ Korea University of Technology and Education \\ Chungnam Province, South Korea
}

Correspondence author email: gwansik@koreatech.ac.kr

\begin{abstract}
TVET trainer plays an important role in ensuring the quality of TVET. TVET Trainer certificate can function as a signal to show the level of their teaching and learning competency. However, only some trainers have TVET trainer certificate. The importance of the role of TVET trainer certificate has recently been emphasized in the Republic of Korea. This study aimed to investigate the effects of the TVET trainers with/without certificate on the training performance. Independent t-tests were conducted to examine the impacts of the trainers on the training performance such as academic achievement, satisfaction level, employment rate and completion rate of trainees. The results showed that TVET trainers with certificate (TWC) tended to be higher in satisfaction level, employment rate, completion rate and academic achievement than TWOCs. On the other hand, the completion rate of trainees learned from TWCs was lower than the result of TWOCs. Based on the results, suggestions for the quality improvement of TVET trainers are discussed.
\end{abstract}

Keywords: Trainer certification; Republic of Korea; TVET trainers; training performance; completion rate DOI: https://10.30880/jtet.2018.10.02.001 


\section{INTRODUCTION}

One of the important preconditions to achieve the objectives and performance related to technical and vocational education and training (TVET) policy is the expertise of the TVET trainers. It is widely believed that trainers are consider as the most important factor, which influences training results (Kim, 2009; Van Driel, Beijaard, \& Verloop, 2001). In that regard, elementary and middle school teachers should acquire educational certificates, which are issued by the government, then pass the teacher employment examination to be a teacher (Kim, 2007).

However, TVET trainers who work for TVET institutions are not required to have a TVET trainer certificate, which is issued by the Ministry of Employment and Labor (MOEL) in Korea. According to the data from the Korean Skills Quality Authority (KSQA), 30,272 TVET trainers applied for the integrated evaluation of business operations on the first half of 2017. Among them, there were only 6,211 TVET trainers with certificate (TWC, 20.5\%), compared to 24,061 TVET trainers without certificate (TWOC, 79.5\%; Korean Skills Quality Authority, 2016). In other words, the number of qualified TVET trainers is substantially insufficient.

Trainer certificate can function as a signal to show the level of teaching and learning competency. TWCs are demonstrating the high quality of training performance, such as effectiveness and efficiency during the teaching and learning processes based on their competency. Furthermore, supplementary trainings are regularly provided for TWCs to support and maintain their sustainable self-development under the rapid transition of job environment. Given that, it might be vital to manage and increase the number of TWCs to improve the quality of training performance at a national level (Cho, Lee, Park, \& Park, 2014; Uh, Cho, \& Kim, 2015).

As the government has recognized the importance of the TWCs, as on effort to improve the quality of TVET since 2017, KSQA sets the certificate status of TVET trainers as one of the relevant evaluation indicators for the approval of training courses (Ministry of Employment and Labor, 2017). Based on the KSQA evaluation indicators, courses operated by TWCs are receive additional merit compared to courses operated by TWOCs. Studies on TVET trainer have been conducted in diverse topics such as qualification standards and the qualification framework (Cho \& Lim, 2010; Cho, Park, \& Lim, 2009; Kim, Lee, \& Cho, 2008; Kim, Kim, Yoon, Lim, Lee, \& Kim, 2004; Lee, Kim, \& Kim, 2008; Oh, Ju, \& Park, 2000), TVET teaching and learning evaluation (Byun, Lee, \& Yoo, 2005; Lee, Byun, \& Kwon, 2011; Lee, Rha, \& Yoo, 2005), national competency standards (Cho, Yoon, \& Park, 2016; Lee, Kim, Kim, Choi, \& Kim, 2016), role and competency of TVET trainers (Choi \& Yoon, 2011; Lee, Lim, \& Kim, 2016; Yoon, 2010), wage of TVET trainers (Sung \& Yoon, 2006), and the actual condition of TVET (Lee et al., 2016; Oh \& Kim, 2005; Oh, Ra, \& Lee, 2008; Uh, Cho, \& Kim, 2015). However, the question of whether there is a difference of training performance between TWCs and TWOCs has not been empirically examined.

In light of that, this study aimed to investigate the utility of current TVET trainer certificate by analyzing and comparing the training performance of TVET trainers with/without TVET certificate. Based on the results, this study suggested relevant implications for the TVET trainers. Research questions for this study are given below: RQ1: Is there any significant mean difference in the trainee satisfaction level and academic achievement depending on whether TVET trainers have been certified or not? RQ2: Is there any significant mean difference in the trainee completion rate and employment rate depending on whether TVET trainers have been certified or not? 


\section{TVET IN REPUBLIC OF KOREA}

\subsection{Characteristics and Competence of TVET Trainers}

TVET trainers in the Republic of Korea are called 'vocational competency development training teachers (TVET trainer)'. "Vocational competency" is the competency which is required to pursue one's duties in a particular or general job. In addition, "vocational competency development" is the educational activities used to acquire and improve the skills for employees and people who have a will to get a job (Korea Society for Learning and Performance, 2010). TVET trainers are in charge of teaching vocational knowledge, skills, and attitudes to the trainees through vocational competency development programmes. TVET trainers in the Republic of Korea cover various work scope since they should carry out the role of teacher for general subjects and take part in some additional roles, such as managing completion and employment of trainees. TVET trainers should be equipped with expertise, competency, know-how in teaching and evaluation, the will to teach students, and sincerity, respect and solicitude for the trainees. The importance of the ability to design, develop and evaluate programmes should also be emphasized (Choi \& Yoon, 2011).

Certificates for TVET trainers consist of 3 levels in total. A person who wants to obtain the certificate must complete the training course for the training of TWCs pursuant to the Minister of Employment and Labor (Workforce Development and Training Act, Article 33, No.13902, 2016). A trainer who works in TVET does not necessarily have to possess a trainer certificate. However, since the legislation was enacted in 2017, incentives are given in the approval of the training courses run by the TWCs. Nowadays, it is mandatory to assign at least 1 TVET trainer by each occupational category of training in the designated TVET institutions. According to the 'workforce development and training act' article 33, TVET trainers or experts in the field who are appointed by a presidential decree could give an instruction in TVET field.

By analyzing the report 'Present conditions of TVET trainer certificate and training market' which was issued by the KSQA in March 2016, the accumulated number of TVET trainers and instructors from the second half of the year 2014 until March 2016 was 45,853. The number of TVET trainers and instructors who registered in the TVET institution registration management system during the integrated evaluation of business operations on the first half of 2017 was 30,272. Among them, only 6,211 persons acquired TVET trainer certificate, which represents $20.5 \%$ of the total number of trainers.

\subsection{TVET performance and the factors of influence}

TVET in Republic of Korea is called 'vocational competency developing and training'. The purpose of the training is to help job seekers and temporary workers to find a job by providing skill training at no cost, and also providing a small amount of training allowance to the beneficiary.

There is a connection between training performance and performance of the training activities. The focus of the performance is always on the trainees. If a trainer gave a successful training, it is linked to the trainee's success. The purpose of TVET is to assist trainees in finding a job, therefore, employment status and wage level are normally used as performance variables. Different from TVET, in the case of business training, productivity is utilized as the key performance indicator.

Lee et al., (2005) conducted a study on the situation of teaching and learning in TVET institutions by collecting data from 756 institutions who are receiving training expense support from the Ministry of Labor. The results showed that the understanding of teaching-learning of TVET institutions was generally high, and they realized that, 'development and management of training 
programmes which address the needs of the field and trainees' was the most important factor. Moreover, 'securing talented trainers' and 'trying effective and various teaching methods' are emphasized (Lee, Rha, \& Yoo, 2005). Maliranta, Nurmi, and Virtanen (2010) analyzed whether there is a significance of student decisions in finding a job or entering school by investigating the characteristics of students in vocational school after compulsory education in Finland as well as the characteristics of vocational schools. As a result, they found that student characteristics such as grade, gender, and family income are influencing the students' decision to find work or to attend to a school of higher level. On the other hand, trainers' academic background was the only factor influencing the students' decision among the characteristics of schools (Maliranta, Nurmi, \& Virtanen, 2010).

Trainers should analyze the teaching objectives by their own point of view and those of the students' to explore the conditions for possibility of learning. Trainers' objectives of interest and human interest are on the activities to acquire competencies, and it is focused on the relationship between trainees and the teaching objective which is the basis of the activities to acquire competencies. Therefore, the trainers' interest is flexible in all aspects of trainee-trainer relationship in terms of situational or processual sides. Trainees require a trainer's support and help, intentional prospect on learning process based on teaching and learning activity, stimulation and feedback, evaluation and review. The subject of the class is the trainee, but the subject of supporting trainees to solve problems in a class is the trainer. The primary precondition to achieve training results, in terms of NCS based competency-based training curriculum and governmental policies, is the expertise of the TVET trainers. It is natural that we recognize trainers as the most important factor which influences educational reform.

\section{METHODOLOGY}

\subsection{Sample and measurements}

This study viewed training performance as a comprehensive term including trainee satisfaction, employment rate, completion rate, and academic achievement. In order to investigate the performance of the TVET trainers, data collection approaches were as follows: first, secondary data managed by Korea Employment Information Service (KEIS) at the national level was used for trainee satisfaction; Second, since employment rate, completion rate, and academic achievement were not accessible or unmanaged at the national level, these data were gathered from training institutions across the nation. Through the process, data were collected from 60 or more courses of each of the representative occupational categories. Thirty or more courses were conducted by TWCs and similar number or more courses were conducted by TWOCs.

In order to assess the trainee satisfaction, the data from KEIS trainee satisfaction surveys were used. The measurements include a total of 9 items, such as 3 items related with trainers, 3 items related with training contents, and 3 items related with training methods. The instrument measuring trainee satisfaction consists of a 5-point Likert scale, ranged from 1 (not at all satisfied) to 5 (very satisfied). The questionnaire surveys on the satisfaction of the training course consisted of the general occupations and Occupations of the National Basic and Strategic Industry (ONBSI). The data were collected from 1,859 courses operated by TWCs among the general occupation courses and 1,415 courses operated by TWOCs. In addition, the data for ONBSI were collected from 1,476 courses operated by TWCs and 1,109 courses operated by TWOCs.

In order to investigate the difference between the academic achievement levels attained by trainers with or without certificate, training courses were selected from three representative occupational categories, which were also approved by KSQA. The academic achievement level is the individual score of the student acquired through summative evaluation at the end of each subject 
(competency unit). The three selected occupational categories were: application software programming in the ICT field, machine element design in the machinery field, and smart culture app content production in the design field. To gather data on the academic achievement of the selected training courses, snowball sampling was implemented among training institutions across the nation. The collection was conducted over a period of 1.5 months starting from September 2017. The collected data includes 127 courses operated by TWCs and 110 courses operated by TWOCs respectively.

To examine the mean difference of the employment rate and the completion rate by trainers with/without TVET certificate, three courses were selected, such as, application software programming in the ICT field, machine element design in the machinery field, and Korean cooking in the service field. The employment rate and the completion rate are calculated as the ratio of the total number of students who have been employed or completed. To collect data on the employment rate and completion rate of the selected training courses, snowball sampling was conducted among training institutions across the nation. The collection was conducted over a period of 2 months starting from July 2017. The data for the employment rate were collected from 192 courses operated by the TWOCs and 225 courses operated by the TWCs. Also, the data for the complement rate were collected from 225 courses operated by TWCs and 192 courses operated by TWOCs.

\subsection{Data Analysis}

The purpose of the study was to examine how TWCs and TWOCs had an impact on TVET performance. In order to investigate whether there was a significant mean difference for each of the TVET performance between TWCs and TWOCs, independent sample t-tests were employed using the SPSS 21 programme. The t-test, which is one of the testing methods of central tendency, is robust to the non-normality of the data, so it is suitable to analyze the ordinal scale as well as interval scale (Murray, 2013; Norman, 2010). In addition, Cohen's d effect size was calculated to examine practical significance, effect size with Cohen's d interpreted by the criterions (i.e., 0.20 as a small effect size; 0.50 as a medium effect size; and 0.80 as a large effect size; Kotrlik \& Williams, 2003).

\section{RESULTS}

\subsection{Trainee satisfaction}

i) Trainee satisfaction (general occupation)

The first part of the trainee satisfaction is consisted of assessing the satisfaction in the field of general occupation. A total of 1,859 courses operated by TWC and a total of 969 courses operated by TWOC were used for the analysis. The impact of trainer on trainee satisfaction was analyzed using independent t-tests. The analysis was grouped into the service field, the ICT field, and the total of the fields. Analysis on the ICT field is excluded since the sample size (minimum 30 samples) was not sufficient. The results of the analysis are shown in Table 1.

Except for the machinery field, t-test results were statistically significant in the service and total. In the service field, TWCs showed higher satisfaction score than TWOCs in the part of trainers, contents of training, and training methods. In addition, the calculated Cohen's d values in the service field ranged from 0.127 to 0.163 , which can be interpreted as not practically meaningful. In the total of fields, the Cohen's d values ranged from 0.095 to 0.157 (less than small effect). 
Table 1: $t$-test results of trainee satisfaction (General occupation)

\begin{tabular}{|c|c|c|c|c|c|c|c|c|}
\hline Field & Area & Status & $\mathbf{N}$ & Mean & SD & $\Delta \mathbf{M}$ & $t$ & Cohen's $d$ \\
\hline \multirow{6}{*}{ Service } & \multirow{2}{*}{ Trainer } & TWC & 1,532 & 4.64 & 0.583 & \multirow{2}{*}{0.104} & \multirow{2}{*}{$4.046^{* * *}$} & 0.163 (less than \\
\hline & & TWOC & 969 & 4.54 & 0.655 & & & small) \\
\hline & \multirow{2}{*}{ Contents } & TWC & 1,532 & 4.49 & 0.686 & \multirow{2}{*}{0.103} & \multirow{2}{*}{$3.446 * * *$} & 0.139 (less than \\
\hline & & TWOC & 969 & 4.39 & 0.756 & & & small) \\
\hline & \multirow{2}{*}{ Method } & TWC & 1,532 & 4.52 & 0.692 & \multirow{2}{*}{0.092} & \multirow{2}{*}{$3.148 * *$} & 0.127 (less than \\
\hline & & TWOC & 969 & 4.43 & 0.732 & & & small) \\
\hline \multirow{6}{*}{ Machinery } & \multirow{2}{*}{ Trainer } & TWC & 312 & 4.58 & 0.640 & \multirow{2}{*}{0.028} & \multirow{2}{*}{0.555} & 0.045 (less than \\
\hline & & TWOC & 368 & 4.55 & 0.677 & & & small) \\
\hline & \multirow{2}{*}{ Contents } & TWC & 312 & 4.42 & 0.747 & \multirow{2}{*}{-0.041} & \multirow{2}{*}{-0.732} & 0.067 (less than \\
\hline & & TWOC & 368 & 4.47 & 0.728 & & & small) \\
\hline & \multirow{2}{*}{ Method } & TWC & 312 & 4.36 & 0.803 & \multirow{2}{*}{-0.102} & \multirow{2}{*}{-1.719} & 0.142 (less than \\
\hline & & TWOC & 368 & 4.47 & 0.746 & & & small) \\
\hline \multirow{6}{*}{ Total } & \multirow{2}{*}{ Trainer } & TWC & 1,859 & 4.63 & 0.595 & \multirow{2}{*}{0.094} & \multirow{2}{*}{$4.180^{* * * *}$} & 0.157 (less than \\
\hline & & TWOC & 1,415 & 4.53 & 0.669 & & & small) \\
\hline & \multirow{2}{*}{ Contents } & TWC & 1,859 & 4.48 & 0.699 & \multirow{2}{*}{0.076} & \multirow{2}{*}{$2.974^{* *}$} & 0.109 (less than \\
\hline & & TWOC & 1,415 & 4.40 & 0.752 & & & small) \\
\hline & \multirow{2}{*}{ Method } & TWC & 1,859 & 4.50 & 0.714 & \multirow{2}{*}{0.068} & \multirow{2}{*}{$2.666^{* *}$} & 0.095 (less than \\
\hline & & TWOC & 1,415 & 4.43 & 0.742 & & & small) \\
\hline
\end{tabular}

\section{ii) Trainee satisfaction (ONBSI)}

The second part of the trainee satisfaction is consisted of assessing the satisfaction in the field of ONBSI. In order to examine the impacts of trainers on trainee satisfaction, independent t-tests were conducted by comparing mean difference of trainee satisfaction between TWCs and TWOCs. The satisfaction of trainee is composed of three parts including trainer, training contents, and training methods. The data was collected from a total of 1,476 courses operated by the TWC and a total of 1,109 courses operated by the TWOCs. The scope of analysis was limited on the ICT and the machinery field. The analysis results are shown in Table 2.

For three areas (trainer, contents, method) in the ICT, the machinery, and the total of the fields, t-test results were statistically significant at the 0.001 level. In other words, it was found that TWCs had a higher score than TWOCs in three areas. In addition, calculated Cohen's d values in the ICT field were ranged from 0.195 (less than small effect) to 0.207 (small to medium effect). In the machinery field, the Cohen's d effect size values ranged from 0.559 to 0.744 (medium to large effect). Lastly, the Cohen's d effect size values in the total of the fields ranged from 0.379 to 0.423 (small to medium effect). 
Table 2: $t$-test results of trainee satisfaction (ONBSI)

\begin{tabular}{|c|c|c|c|c|c|c|c|c|}
\hline Field & Area & Status & $\mathbf{N}$ & Mean & SD & $\Delta \mathbf{M}$ & $t$ & Cohen's $d$ \\
\hline \multirow{6}{*}{ ICT } & \multirow{2}{*}{ Trainer } & TWC & 660 & 4.37 & 0.901 & \multirow{2}{*}{0.197} & \multirow{2}{*}{$4.005^{* * *}$} & 0.207 (small to \\
\hline & & TWOC & 861 & 4.17 & 1.013 & & & medium) \\
\hline & \multirow{2}{*}{ Contents } & TWC & 660 & 4.20 & 0.954 & \multirow{2}{*}{0.191} & \multirow{2}{*}{$3.681^{* * *}$} & 0.195 (less than \\
\hline & & TWOC & 861 & 4.00 & 1.069 & & & small) \\
\hline & \multirow{2}{*}{ Method } & TWC & 660 & 4.16 & 1.006 & \multirow{2}{*}{0.211} & \multirow{2}{*}{$3.870^{* * *}$} & 0.196 (less than \\
\hline & & TWOC & 861 & 3.95 & 1.117 & & & small) \\
\hline \multirow{6}{*}{ Machinery } & \multirow{2}{*}{ Trainer } & TWC & 816 & 4.62 & 0.673 & \multirow{2}{*}{0.433} & \multirow{2}{*}{$6.266^{* * *}$} & 0.559 (medium to \\
\hline & & TWOC & 248 & 4.19 & 1.023 & & & large) \\
\hline & \multirow{2}{*}{ Contents } & TWC & 816 & 4.48 & 0.769 & \multirow{2}{*}{0.603} & \multirow{2}{*}{$7.870^{* * *}$} & 0.692 (medium to \\
\hline & & TWOC & 248 & 3.88 & 1.131 & & & large) \\
\hline & \multirow{2}{*}{ Method } & TWC & 816 & 4.47 & 0.806 & \multirow{2}{*}{0.678} & \multirow{2}{*}{$8.345^{* * *}$} & 0.744 (medium to \\
\hline & & TWOC & 248 & 3.79 & 1.201 & & & large) \\
\hline \multirow{6}{*}{ Total } & \multirow{2}{*}{ Trainer } & TWC & 1,476 & 4.51 & 0.793 & \multirow{2}{*}{0.333} & \multirow{2}{*}{$9.070^{* * *}$} & 0.379 (small to \\
\hline & & TWOC & 1,109 & 4.17 & 1.015 & & & medium) \\
\hline & \multirow{2}{*}{ Contents } & TWC & 1,476 & 4.35 & 0.868 & \multirow{2}{*}{0.378} & \multirow{2}{*}{$9.546^{* * *}$} & 0.382 (small to \\
\hline & & TWOC & 1,109 & 3.98 & 1.084 & & & medium) \\
\hline & \multirow{2}{*}{ Method } & TWC & 1,476 & 4.33 & 0.914 & \multirow{2}{*}{0.417} & \multirow{2}{*}{$\begin{array}{c}10.026^{*} \\
* *\end{array}$} & 0.423 (small to \\
\hline & & TWOC & 1,109 & 3.91 & 1.138 & & & medium) \\
\hline
\end{tabular}

\subsection{Trainee academic achievement}

In order to examine the effects of trainers on academic achievement, independent sample t-tests were conducted in four parts including the ICT, the design, the machinery, and the total of the fields. The data were collected from a total of 127 courses operated by the TWCs and a total of 110 courses operated by the TWOCs. The analysis results are shown in Table 3.

Table 3: $t$-test results of academic achievement

\begin{tabular}{|c|c|c|c|c|c|c|c|}
\hline Field & Status & $\mathbf{N}$ & Mean & SD & $\Delta \mathbf{M}$ & $t$ & Cohen's d \\
\hline \multirow{2}{*}{ ICT } & TWC & 31 & 84.68 & 4.219 & \multirow{2}{*}{3.723} & \multirow{2}{*}{$3.224^{* *}$} & \multirow{2}{*}{0.802 (large) } \\
\hline & TWOC & 36 & 80.95 & 5.098 & & & \\
\hline \multirow{2}{*}{ Machinery } & TWC & 52 & 84.15 & 5.074 & \multirow{2}{*}{1.679} & \multirow{2}{*}{1.667} & 0.341 (cannot be \\
\hline & TWOC & 43 & 82.48 & 4.652 & & & interpretable) \\
\hline \multirow{2}{*}{ Service } & TWC & 44 & 82.06 & 5.351 & - & \multirow{2}{*}{-0.405} & \multirow{2}{*}{0.099 (less than small) } \\
\hline & TWOC & 31 & 82.66 & 6.890 & 0.598 & & \\
\hline \multirow{2}{*}{ Total } & TWC & 127 & 83.56 & 5.067 & \multirow{2}{*}{1.527} & \multirow[t]{2}{*}{$2.223^{*}$} & 0.290 (small to \\
\hline & TWOC & 110 & 82.03 & 5.507 & & & medium) \\
\hline
\end{tabular}

In the ICT field, t-test results showed that TWCs were higher than TWCOs, and the mean differences were statistically significant. However, there was no statistically significant mean difference in the design and the machinery fields. On the other hands, t-test results were statistically 
significant at the 0.01 level in the total of the fields. Calculated Cohen's $d$ value in the ICT field was 0.802 (large effect), and the Cohen's d value in the total part was 0.290 (small to medium effect).

\subsection{Trainee completion rate}

To examine the impact of trainer on trainee completion rate, independent t-tests were conducted by comparing mean difference of trainee completion rate between TWCs and TWOCs. As earlier, three fields of training data were used including the ICT, the machinery, and the service field. The data was collected from a total of 225 courses operated by the TWC and a total of 192 courses operated by the TWOCs. The analysis results are shown in Table 4.

For three fields including the ICT, the machinery, and the total, t-test results were statistically significant at the 0.001 level except service field. Although the t-test result in the service field was not statistically significant, the Cohen's d value was 0.338 , which cannot be interpretable. Also, it was found that TWCs had a lower score than TWOCs in three fields. In addition, calculated Cohen's d value in the ICT field was 0.627 (medium to large effect), the Cohen's d value in the machinery field was 0.654 (medium to large effect), lastly, the Cohen's d value in the total of the fields was 0.534 (medium to large effect).

Table 4: $t$-test results of completion rate (\%)

\begin{tabular}{|c|c|c|c|c|c|c|c|}
\hline Field & Status & $\mathbf{N}$ & Mean & SD & $\Delta \mathbf{M}$ & $t$ & Cohen's $d$ \\
\hline \multirow{2}{*}{ ICT } & TWC & 79 & 72.46 & 15.121 & \multirow{2}{*}{-8.972} & - & \multirow{2}{*}{0.627 (medium to large) } \\
\hline & TWOC & 78 & 81.43 & 13.415 & & $3.934^{* * *}$ & \\
\hline \multirow{2}{*}{ Machinery } & TWC & 90 & 76.80 & 21.655 & \multirow{2}{*}{-12.271} & - & \multirow{2}{*}{0.654 (medium to large) } \\
\hline & TWOC & 56 & 89.08 & 12.868 & & $4.294^{* * *}$ & \\
\hline \multirow{2}{*}{ Service } & TWC & 56 & 89.08 & 18.920 & \multirow{2}{*}{-4.867} & \multirow{2}{*}{-1.804} & \multirow{2}{*}{$\begin{array}{c}0.338 \text { (cannot be } \\
\text { interpretable) }\end{array}$} \\
\hline & TWOC & 58 & 93.95 & 7.873 & & & \\
\hline \multirow{2}{*}{ Total } & TWC & 225 & 78.33 & 19.895 & \multirow{2}{*}{-9.107} & - & \multirow{2}{*}{0.534 (medium to large) } \\
\hline & TWOC & 192 & 87.44 & 12.927 & & $5.438^{* * *}$ & \\
\hline
\end{tabular}

\subsection{Trainee employment rate}

In order to examine the impact of trainer on trainee employment rate, independent t-tests were conducted by comparing mean difference of trainee employment rate between TWCs and TWOCs. Three fields of training data were used including the ICT, the machinery, and the service field. The data were collected from a total of 225 courses operated by the TWC and a total of 192 courses operated by the TWOCs. The analysis results are shown in Table 5.

For three parts including the ICT, machinery, and total of the fields, t-test results were statistically significant at the 0.01 level or above. In other words, it was found that TWCs had a higher score than TWOCs in three fields. In addition, calculated Cohen's d value in the ICT field was 0.464 (small to medium effect), the Cohen's d value in the machinery area was 1.013 (large effect), and the Cohen's d value of the service field was 0.445 (small to medium effect), lastly, the Cohen's d value in total of the fields was 0.612 (medium to large effect). 
Table 5: $t$-test results of employment rate (\%)

\begin{tabular}{|c|c|c|c|c|c|c|c|}
\hline Field & Status & $\mathbf{N}$ & Mean & SD & $\Delta \mathbf{M}$ & $t$ & Cohen's $d$ \\
\hline \multirow{2}{*}{ ICT } & TWC & 79 & 62.61 & 15.666 & \multirow{2}{*}{8.295} & \multirow{2}{*}{$2.899^{* *}$} & \multirow{2}{*}{0.464 (small to medium) } \\
\hline & TWOC & 78 & 54.31 & 19.908 & & & \\
\hline \multirow{2}{*}{ Machinery } & TWC & 90 & 62.45 & 21.880 & \multirow{2}{*}{21.451} & \multirow{2}{*}{$5.949^{* * *}$} & \multirow{2}{*}{1.013 (large) } \\
\hline & TWOC & 56 & 41.00 & 20.009 & & & \\
\hline \multirow{2}{*}{ Service } & TWC & 56 & 37.75 & 16.296 & \multirow{2}{*}{8.277} & \multirow{2}{*}{$2.384^{*}$} & \multirow{2}{*}{0.445 (small to medium) } \\
\hline & TWOC & 58 & 29.47 & 20.591 & & & \\
\hline \multirow{2}{*}{ Total } & TWC & 225 & 56.36 & 21.358 & \multirow{2}{*}{13.433} & \multirow{2}{*}{$6.205^{* * *}$} & \multirow{2}{*}{0.612 (medium to large) } \\
\hline & TWOC & 192 & 42.93 & 22.597 & & & \\
\hline
\end{tabular}

$-* p<.05, * * p<.01, * * * p<.001$

- note: TWC (Trainers with Certificate), TWOC (Trainers without Certificate)

5

DISCUSSION

Based on the result, this study could provide several relevant suggestions to improve the performance of TVET, encouraging expertise of TWCs and TWOCs. First, in case of trainee satisfaction, the results were statistically significant but not practically meaningful in the general occupation. On the other hand, those values were statistically and practically significant in ONBSI except for some fields. One of possible interpretation for the results might be due to occupational characteristics. The educational objectives are specific and the level of difficulty tends to be simple in general occupations since trainings are short (more than 30 hours). Compared to this, training courses in ONBSI should be conducted for 350 hours with 3 months or more. Considering these circumstances, TWCs might be better at managing the long term courses, and delivering specialized knowledge step by step. In this regards, it is conceivable that the importance of TWCs could be emphasized, specifically in ONBSI field. In addition, various plans to increase the number of TWCs should be reviewed to improve the quality of training in the long run, since the ratio of TWC is still small among total TVET trainer in Korea (Korean Skills Quality Authority, 2016).

Second, the results showed that the overall employment rate was higher in TWCs, while the overall completion rate was higher in TWOCs. Since the final goal of trainees is employment (Oh \& Kim, 2005), we need to focus on the result that the employment rate was higher when the trainees learned from TWCs. It might be interpreted that TWCs tend to be conservative about the completion criteria by considering future employability or capability of trainees. However, additional quantitative and qualitative studies should be conducted to understand this phenomenon more deeply.

Third, it is vital to consider other performance variables which could identify performance of TVET more comprehensively. Currently, types of quantitative indicators used to measure the performance factors in TVET such as trainee academic achievement level, trainee satisfaction level with trainers or training environment, completion rate, employment rate, certificate acquisition, and ratio of employment maintenance (Lee et al., 2016), are very limited. Given that, the results of performance indicators (e.g., competencies, soft-skills, quality of employment) utilized in this study needs to be further examined and discussed to enhance the quality of TVET. In addition, more performance indicators need to be identified and examined.

Lastly, all of data were collected directly except the satisfaction data for this study, since there is no national data collection and management system in the TVET field. Also, training institutions were reluctant to share their performance-related data. Considering that, MOEL and KEIS need to pay 
more attention to establishing and managing the national-level TVET performance database system. The performance data should be managed continuously for the quality improvement of TVET training based on TWCs as well as for the performance management of TWCs through big data.

\section{CONCLUSION}

TVET trainers could play the important role of supporting trainees to enhance their competencies, as well as employability. The importance of TWCs has recently been emphasized in the Republic of Korea. In this regards, this study aimed to investigate the utility of current TVET trainer certificate by analyzing and comparing the training performance of TVET trainers with/without TVET certificate. The results of statistical analysis to examine the difference of the variables such as satisfaction level, employment rate, completion rate and academic achievement of trainees between TVET trainers with/without TVET certificate are given below.

First, according to the analysis results of trainee satisfaction, there is a statistically significant mean difference regarding trainer, training contents and training method in the service and the total of the fields among general occupation courses. However, the Cohen's d value showed that there is a less than small effect size. In case of t-test, it is able to be significant when the sample gets bigger. There is a very limited practical effect in the difference between TWCs and TWOCs, if the effect of sample size is exempted. Regarding the machinery field, there is no difference in the t-test result and the Cohen's d value. In terms of ONBSI courses, there is a statistically significant mean difference in training contents and training method of the ICT sector and also a limited practical effect. On the other hand, the analysis results on trainer and the total of the fields showed that there is a statistically significant mean difference as well as a small to medium size effect which is practically meaningful. In the machinery field, the results showed that there is a statistically and practically significant difference in trainer, training contents and training method. To sum up, the difference of trainee satisfaction between the courses given by TWC and TWOC in general occupation was very limited, however in case of ONBSI courses, trainee satisfaction was higher in courses given by TWCs compared to the courses delivered by TWOCs.

Second, when it comes to academic achievement, the results supported that there is a statistically and practically significant mean difference in the ICT and the total of the field. That is to say that the academic achievement of TWC led courses is higher than TWOC led courses. The results of the machinery field showed that there is no statistically significant mean difference, but we found that there is a small to medium size effect by applying Cohen's $d$ value analysis. In case of the service sector, the results showed that there is no practically and statistically significant difference.

Third, in terms of completion rate, the analysis results showed that there is a statistically and practically significant mean difference in the ICT, the machinery and the total of the field. Therefore, the completion rate of the courses delivered by TWCs was higher than those of TWOCs. When it comes to the service field, it is hard to say that the statistically significant mean difference exists, but interestingly, Cohen's d supported that there is a small to medium effect size. So it is better to be analyzed more specifically in further studies.

Fourth, in case of employment rate, all of the results showed that there is a statistically and practically significant mean difference in the ICT, the machinery, the service and the total of the field. In other words, employment rate of the courses given by TWCs was higher than the courses by TWOCs. To sum up, we found a tendency that trainee satisfaction, academic achievement and employment rate of courses managed by TWC were mostly higher compared to courses led by TWOC. Based on the results of the study, this study could make a positive contribution to the existing 
literature by providing the initial empirical evidence to examine training performance of TVET trainers with/without certificate in Korean context.

\section{LIMITATIONS AND RECOMMENDATIONS}

The limitations of this study are as follows. First, due to limitations in data collection, this study collected and analyzed the data with convenient sample approach. It needs to understand the results by considering the characteristics of the sample and interpret the results cautiously, so as not to generalize the results to all types of occupation and training courses. Given that, future studies need to be replicated to diverse types of occupation and training courses. Second, this study conceptualized the performance of TVET with employment rate, completion rate, academic achievement, and satisfaction level. Also, regarding the characteristics of trainers, this study only considered whether or not trainers possess TVET certificate, however, it did not consider other potentially meaningful factors such as the expertise (capacity) of trainers, level of trainer certificate, or acquisition path of the certificate. Considering them, it might be more helpful to specifically and accurately examine TVET performance between TWCs and TWOCs. Empirical follow-up studies to analyze the effects of various TVET trainer characteristics on TVET performance are also encouraged.

\section{References}

Byun, S., Lee, S., \& Yoo, S. (2005). Teaching-learning Improvement Method In Vocational Training(II) (Report No. 2005-07). Seoul: KRIVET.

Cho, J., \& Lim, K. (2010). Korean qualification framework (KQF) establishment plan. THE HRD REVIEW, 13(3), 65-96.

Cho, J., Park, D., \& Lim, K. (2009). Construction of Korean Skills Standards and National Qualification System (2009) : Internationalization of Qualification (Report No. 09-08-10). Seoul: KRIVET.

Cho, S., Lee, C., Park, B., \& Park, J. (2014). A Study on Competency Modeling and Programme Development of Vocational Competency Development Training Teachers. Cheonan: Korea University of Technology and Education.

Cho, S., Yoon, D., \& Park, J. (2016). Research on NCS and Course Based Qualification Operation Situation and Its Performance Analysis (Report No. 2015-19). Ulsan: HRD Korea.

Choi, M., \& Yoon, G. (2011). A Study of The Role and Competencies of Vocational Training Teachers: Focusing on Public Institution. The Journal of Vocational Education Research,30 (2), 265-294.

Kim, C. (2009). In-service Education and Development of Professional Teaching Ability. The Journal of Educational Research and Practice, 75, 67-90.

Kim, H., Lee, D., \& Cho, J. (2008). Development of NCS and Establishment of Qualification Framework: Establishment of KVQ based on NCS. Seoul: KRIVET.

Kim, J., Kim, B., Yoon, G., Lim, S., Lee, S., \& Kim, S. (2004). Improvement Plan of TVET Trainer Qualification Standard (Report No. 2004-02). Seoul: KRIVET.

Kim, S. (2007). A Clarification and Institutionalization of Requirements for Certificate of Teachers. The Journal of Yeolin Education, 15 (3), 27-53.

Korea Society for Learning and Performance. (2010). Dictionary of HRD Terms. Seoul: Joongang Economic Daily.

Korean Skills Quality Authority. (2016). Present Conditions of TVET Trainer Certificate and Training Market. Informally published work.

Kotrlik, J. W., \& Williams, H. A. (2003). The Incorporation of Effect Size in Information Technology, Learning, and Performance Research. Information Technology, Learning, and Performance Journal,21 (1), 1-7.

Lee, D., Kim, H., \& Kim, H. (2008). Development of Korean Skills Standards and Construction of Qualification System (2008): Development of Job Classification in Food Processing and Cooking Sector (Report No. 0840-14). Seoul: KRIVET.

Lee, J., Kim, Y., Kim, J., Choi, G., \& Kim, J. (2016). Research on Improvement of Labor Conditions of TVET Trainers. Cheonan: Korea University of Technology and Education HRD Center.

Lee, M., Lim, S., \& Kim, W. (2016). A Need Analysis on Roles of Teachers That Trainees Perceive in Private Vocational Training Institutes. Journal of Competency Development \& Learning,11 (2), 57-78. 
Lee, S., Byun, S., \& Kwon, S. (2011). Teaching Method for Strengthening Vocational Training Competitiveness-study (Report No. 11-13). Seoul: KRIVET.

Lee, S., Min, S., Moon, S., Jo, S., \& Kim, G. (2016). Result Analysis on Application and Proliferation of NCS. Ulsan: HRD Korea.

Lee, S., Rha, H., \& Yoo, S. (2005). Analyze The Present Status of Teaching and Learning at Vocational Training Institutions. The Journal of Vocational Education Research, 24 (1), 173-191.

Maliranta, M., Nurmi, S., \& Virtanen, H. (2010). Resources in Vocational Education and Post-schooling Outcomes. International Journal of Manpower, 31 (5), 520-544.

Ministry of Employment and Labor. (2017). Notice of Ministry of Employment and Labor 2017-20 Vocational Competency Development Training Teacher Qualification Standard.

Murray, J. (2013). Likert Data: What to Use, Parametric or Non-Parametric?. International Journal of Business and Social Science, 4 (11), 258-264.

Norman, G. (2010). Likert Scales, Levels of Measurement and The "Laws" of Statistics. Adv in health Sci Educ, 15, 625-632.

Oh, Y., Ju, I., \& Park, D. (2000). The Improvement of Qualification System in Vocational Training Instructor (Report No. 2000-33). Seoul: KRIVET.

Oh, Y., \& Kim, S. (2005). Characteristics and Structure of the Vocational Training Market (2): Analysis of Vocational Training Institutes and Procedural Structure (Report No. 05-08-02). Seoul: KRIVET.

Oh, Y., Ra, Y., \& Lee, S. (2008). Employment Condition of TVET Trainer and Policy Improvement Plan (Report No. 2008-35). Seoul: KRIVET.

Sung, J., \& Yoon, S. (2006). Determinants of Vocational Training Teachers Wage. Korean Journal of Labor Economics, 29 (2), 177-199.

Uh, S., Cho, S., \& Kim, D. (2015). A Study on the Improvement of Qualification Standard System for Teachers of Technology and Vocational Education and Training. Cheonan: Korea University of Technology and Education.

Van Driel, J. H., Beijaard, D., \& Verloop, N. (2001). Professional Development and Reform in Science Education: The Role of Teachers' Practical Knowledge. Journal of Research in Science Teaching, 38 (2), 137-158.

Workforce Development and Training Act, Article 33, No.13902 (2016).

Yoon, G. (2010). The Roles and Job Analysis on the Vocational Training Instructors of Private Vocational Training Schools. The Journal of Vocational Education Research, 29 (4), 149-163. 